\title{
Inaugural Statement
}

Received 05 March, 2021

Accepted 27 April 202

Zhangsuo Liu

Department of Nephrology, the First Affiliated Hospital of Zhengzhou University; Research Institute of Nephrology, Zhengzhou University, Zhengzhou, Henan Province, China

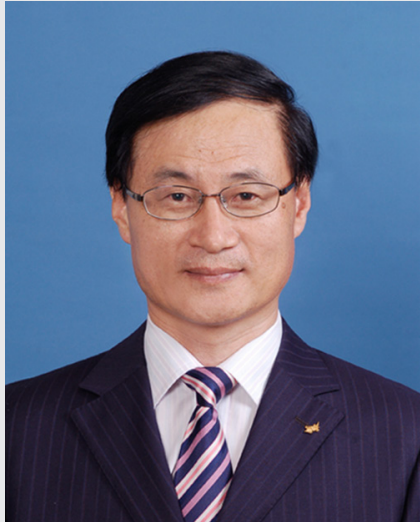

Liu Zhangsuo is a Professor, Chief physician, and Ph.D. supervisor. $\mathrm{He}$ is associated with Central Plains Scholars, national outstanding scientific and technological workers, Special government allowances of the State Council, young and middle-aged experts with outstanding contributions to national health and family planning. $\mathrm{He}$ is also the current Vice President of Zhengzhou University, Dean of the First Affiliated Hospital of Zhengzhou University, Director of the Research Institute of Nephrology, Zhengzhou University, Director of Henan Nephrology Research Center, Director of Henan Provincial Key Laboratory of Precision Diagnosis and Treatment of Chronic Kidney Disease, Henan Clinical Medicine Research on Nephropathy Director of the Centre. He also holds the distinction of being the Vice-chairman of the expert committee of kidney disease, capacity building and continuing education center, National Health Commission, a Standing Committee Member and Secretary-General of Chinese Society Nephrology, Designate Chairman of Chinese Society of Medical Information, and Chairman of Nephrology Professional Committee of Chinese Research Hospital Association and also holds other positions.
Diabetic Nephropathy, with high prevalence, is the main cause of renal failure in diabetic patients. It is urgent to strengthen the prevention and treatment of diabetic nephropathy. As Diabetic Nephropathy launches its first issue, we explain our original intention of setting up the journal and outline the aims and dreams what we want to achieve. We welcome you, the readers, authors, and referees, to join us on the journey in pursuit of knowledge.

According to IDF Diabetes Atlas 9th edition by World Health Organization, the total number of patients with Diabetes Mellitus (DM) is 463 million in 2019, which means 1 in 11 people is diabetic. Diabetic nephropathy is one of the most common and severe microvascular complications of DM, and its incidence is $>50 \%$ [1]. Therefore, it is urgent to strengthen the prevention and treatment of diabetic nephropathy. Nowadays, more and more basic research and scientific research projects focus on the field of diabetic nephropathy, and the quantity and quality of papers published in the international community are increasingly noticeable. However, there are no Science Citation Index (SCI) journals in this field worldwide. Thus, the journal of Diabetic nephropathy $(D N)$ is being launched this year.

As the Editor-in-Chief of $D N$, our goals, of course, are not just to add another journal to enrich an already extended list of open access publications. What's more, we also hope to construct a bridge between Chinese and World scientific and clinical research on diabetic nephropathy. According to the latest epidemiological data of diabetes and pre-diabetes published by JAMA in 2013, the prevalence rate of adult diabetes was $10.9 \%$, whereas the prevalence rate of prediabetes was $35.7 \%$ in China. Among the people receiving treatment, only less than half of the patients $(49.2 \%)$ had their HBA1c level effectively controlled $(<7.0 \%)$ [2]. With such a large number of diabetic people and such a high incidence of kidney involvement, it can be predicted that in the next 5-10 years, diabetic nephropathy in China will inevitably become the primary cause of chronic kidney disease. During the last decade, growing research activities have been conducted to explore the mechanism of diabetic nephropathy progression and treatment strategies by Chinese physicians and researchers. The journal of $D N$ will provide the scientific community of China with an urgently needed outlet to present its latest scientific achievements to the international community.

This is an era of thriving biomedical research. With an in-depth study of exosomes, stem cells, and epigenetic modifications, we can better reveal the pathogenesis of diabetic nephropathy. With the development of renal biopsy techniques, we can better distinguish diabetic nephropathy from 
diabetic kidney disease, which will guide clinical therapeutic regimen. With further research of novel therapeutic drugs, we can constantly update the clinical guidelines. Undoubtedly, the understanding of diabetic nephropathy is deepening. However, we cannot and must not stop there. Science continually advances, and to fully appreciate the complicated pathogenesis of diabetic nephropathy and even struggle to conquer it, so must nephrologist.

To get a much more refined understanding of the disease, nephrologists urgently need a convergence to access professional information and showcase their latest academic progress. It is for this reason that the journal of $D N$ is being launched. We are aiming at establishing an English medical journal that can lead the world in the field of diabetic nephropathy and related basic medicine, clinical medicine, and general health, and show its latest academic progress. What's more, we will publicize journals and invite high-level papers at home and abroad through the Internet and conferences which ensures that we receive contributions from other countries or regions.

As for publishing patterns, we will adopt the world's popular Open Access publishing model to provide free downloads to global readers and enter the PubMed Central system in a short time to improve the international exhibition. Our professional publisher, Elsevier Publishing, provides the internationally popular manuscript review platform (Editorial Manager). At the same time, the journal will support online Early Publication of Epub Ahead of Print. As English is the first language and the primary language for academic writing in the world, and as this journal being an international magazine, the contributions will be accepted in the English language. In conclusion, we will strictly abide by international editing and publishing norms and strategies, and start and run periodicals conforming to international high-level periodical standards.

With this inaugural issue, we proudly announce the birth of Diabetic Nephropathy. In the first issue, we offer a selection of articles that represent our broad remit. From the study of endoplasmic reticulum stress in diabetic nephropathy and a review of a potential therapeutic target for diabetic nephropathy to the exploration of the effect of diabetes on protein-energy wasting in the maintenance of hemodialysis patients, our scope is all-encompassing but targeted.

It is only the beginning now, and we will do our very best though we have a long way to go. However, with continuing support from international colleagues, we strongly believe that Diabetic Nephropathy will be a valuable and highly appreciated source of information and knowledge for clinicians and scientists worldwide. We would love to hear from you and a hearty welcome to our readers.

\section{REFERENCES}

[1] International Diabetes Federation. IDF Diabetes Atlas. 9th ed. International Diabetes Federation, Brussels, Belgium 2019.

[2] Xu Y, Wang L, He J, Bi Y, Li M, Wang T, et al. Prevalence and control of diabetes in Chinese adults. JAMA 2013; 310:948-59. 